\title{
The Prospects for the Therapeutic Implications of Genetically Engineered Probiotics
}

\author{
Sayyeda Farwa Mazhar, ${ }^{1}$ Muhammad Afzal, ${ }^{1}$ Ahmad Almatroudi $\mathbb{D}^{2},{ }^{2}$ Samman Munir, ${ }^{3}$ \\ Usman Ali Ashfaq, ${ }^{3}$ Maria Rasool, ${ }^{1,4}$ Hammad Raza, ${ }^{5}$ Hafiz Muhammad Waqas Munir, ${ }^{1}$ \\ Muhammad Shahid Riaz Rajoka, ${ }^{6}$ and Mohsin Khurshid $\mathbb{D}^{4}$ \\ ${ }^{1}$ College of Allied Health Professionals, Directorate of Medical Sciences, Government College University Faisalabad, \\ Faisalabad, Pakistan \\ ${ }^{2}$ Department of Medical Laboratories, College of Applied Medical Sciences, Qassim University, Qassim 51431, Saudi Arabia \\ ${ }^{3}$ Department of Bioinformatics \& Biotechnology, Government College University Faisalabad, Faisalabad, Pakistan \\ ${ }^{4}$ Department of Microbiology, Government College University Faisalabad, Faisalabad, Pakistan \\ ${ }^{5}$ Department of Biochemistry, Government College University Faisalabad, Faisalabad, Pakistan \\ ${ }^{6}$ College of Chemistry and Environmental Engineering, Shenzhen University, Shenzhen 518060, Guangdong, China
}

Correspondence should be addressed to Mohsin Khurshid; mohsin.mic@gmail.com

Received 3 January 2020; Revised 7 March 2020; Accepted 18 March 2020; Published 7 April 2020

Academic Editor: Efstathios Giaouris

Copyright (C) 2020 Sayyeda Farwa Mazhar et al. This is an open access article distributed under the Creative Commons Attribution License, which permits unrestricted use, distribution, and reproduction in any medium, provided the original work is properly cited.

\begin{abstract}
The interest in the therapeutic use of probiotic microorganisms has been increased during the last decade although the doubts have ascended about the probiotics mainly because their beneficial effects are not fully understood, and, in many cases, their usefulness has not been validated in clinical trials. Consequently, the notion got a considerable interest in those strains having proven probiotic potential to be engineered for improvement in their beneficial features. The process of genetic engineering can also be used for probiotic strains for the reversion of antimicrobial resistance and other modifications for their safer and effective human applications. The lactic acid bacilli are predominantly opposite as they already have gained attention owing to their healthpromoting benefits and their safety for human consumption; therefore, their use, especially as a delivery agent of vaccines and drugs, is gaining attention. The tailoring of probiotic strains will not only improve the data regarding the probiotic potential of these strains but also clinch the doubts concerning these probiotics. This article focuses on the approaches of bioengineered probiotics and discusses the potential prospects for their therapeutic applications including immunomodulation, cognitive health, and anticancer therapeutics.
\end{abstract}

\section{Introduction}

Probiotic is a Greek word; "pro" means "in favor' and "biotic" means "life." Probiotics are living microbes harboring useful properties for the host largely through the balancing of intestinal microbiota [1-3]. The therapeutic use of probiotics has found to be helpful for both humans and animals for prophylaxis and therapeutic purposes. Although the intake of beneficial microorganisms by human beings for health, food, and nutrition is practiced from centuries, however, the precise requirement of probiotics bacteria for the provision of positive health effects may vary from strain to strain; the $10^{6}$ live bacteria per gram or milliliter of the food product is the lowest level that is considered sufficient [4]. There are numerous potential benefits of probiotics, for example, metabolic modulation, inhibition of colonization of opportunistic pathogens in the intestine, lactose in-tolerance reduction, and maintenance of a balance of gut microflora. Conversely, lactic acid bacilli are responsible for spoilage of processed meats which results in considerable financial losses for the food-producing companies [5] as well as responsible for infections including endocarditis, 
bacteremia, and others in susceptible patients [6]. Some enterococci were found to harbor virulence factors including adhesins, invasins, and hemolysins as well as antibioticresistant determinants [7-9].

Although the host responses to the infectious microbes are thoroughly studied, the major concern is to explore safe and novel therapies using the beneficial microorganisms [10-13]. The production of novel bioengineered probiotics can be accomplished through genetic modifications which will not only enhance the efficacy of conventional probiotics but also lessen the pathogenic potential of these strains. Moreover, these strains can be used for a few additional applications such as a vaccine or drug delivery, mimicking surface receptors, targeting specific toxins or pathogens, and enhancement of host immune responses [14]. The genetic engineering of formerly nonprobiotic strains to get probiotic characteristics and the enhancement of probiotic properties of established probiotic strains are the ongoing approaches to design and develop new genetically modified probiotics $[15,16]$.

Although the probiotics were primarily used for the improvement of human health, the formulations of a few well-characterized strains such as Bifidobacteria and lactic acid bacteria (LAB) are also available nowadays for human use to lower the risk of gastrointestinal infections. Further, a strong relationship is suggested between the probiotics and the immune-modulatory responses in humans [17]. To design the novel probiotics with desired features, a detailed study of the limitations of conventional probiotics is necessary $[18,19]$. The various aspects of genetically modified (GM) probiotics have now got considerable acceptance especially related to their potential to fill the present gaps in their spectrum of activity as a probiotic. Therefore, it is anticipated that the careful design of GM probiotics with complete attention to their biological safety has the significant potential to transform the microbial-based therapeutics. In the present study, we have described the current status and limitations of the conventional probiotics and the need of the engineered probiotic strains. Moreover, the utility of designer probiotics as therapeutic agents for the treatment of various noncommunicable diseases as well as infectious diseases through the production of antimicrobial peptides has been discussed. Further, the safety concerns and regulatory issues of the genetically modified probiotic strain in the clinical settings are also described.

\section{Conventional Probiotics and Their Limitations}

Gastrointestinal tract (GIT) microflora differs among individuals and contains both pathogenic and friendly bacteria existing in a symbiotic relation. Several factors such as diet, aging climate, medication, lifestyle, and stress can disturb the balance situation between microbial species and can lead to illnesses [20]. Although probiotics have numerous benefits in GIT conditions, there are certain limitations as well. The antimicrobial substances that are released by probiotics have a broad range, but the studies have shown that the antibacterial activity of probiotics is limited to particular microbes [21, 22]. So the combination of several probiotic strains should be produced to augment the effects against pathogens within the intestine [23]. Most probiotics are administered as capsules or as a food component; therefore, they should have the ability to resist both gastrointestinal and technological stress. A wide range of activities and the differences between the different probiotic strains are also considered as a barrier in their efficacy [24]. Besides, the precise constituents of probiotic formulations, dosage, and the route of administration may vary, and their probiotic potential can differ in certain hosts [25].

The main concern for most probiotics is the survival of probiotic strains in the food products during their exposure to low $\mathrm{pH}$ following fermentation, oxygen concentration during refrigeration process, and storage and their viability during their stay in the human stomach due to the acidic environment. This survival and viability of probiotics are strain-specific; therefore, the microencapsulation procedures have been effectively used for the protection of bacterial cells from the damages induced by their external environment $[26,27]$. Further, the challenges include the sensory acceptance of probiotic-based foods although the studies have described the likelihood of obtaining comparable or even better results with probiotic-based products compared with the conventional products. Moreover, the further challenges include inoculum size, the evaluation of viable counts of particular probiotic species especially when multiple strains are used, the origin and diversity of probiotics, and survival as well as interaction with the endogenous microflora and provision of health benefits to the host [17]. The limitations of conventional probiotics emphasize the need to develop a new strategy to produce GM probiotic strains [28].

\section{Mechanism of Action of Probiotics}

Establishment of host-microbial relationships is imperative for the host health and the disturbances of such interactions in the GIT may lead to numerous pathological conditions $[29,30]$. To maintain such microbial balance within the GI tract, the probiotics need to competitively inhibit the adherence of pathogens to GI tract epithelium as well as to synthesize or produce new novel antimicrobial substances. Probiotics have been shown to confer direct inhibitory effects on pathogens by producing substances like bacteriocins and acids [30]. The antimicrobial molecules derived from probiotics exert their useful effects either alone or synergistically to control the growth of pathogens. Moreover, the probiotic strains such as lactobacilli are well recognized for producing lactic, acetic, and propionic acids that decrease the $\mathrm{pH}$, therefore inhibiting a vast range of pathogenic particularly Gram-negative bacteria [31, 32]. Further, the probiotics inhibit the toxin release as well as inhibit the adhesion of pathogenic bacteria within the GI tract. Bifidobacteria and lactobacilli strains are capable of competing with pathogenic microorganisms, including Bacteroides vulgatus, Clostridium histolyticum, Listeria monocytogenes, Yersinia enterocolitica, Staphylococcus aureus, 
enterotoxigenic E. coli, and Salmonella enterica in the gut epithelium before any probiotic treatment $[33,34]$.

The integrity of the intestinal barrier is a precondition for an effective mucosal function to maximize the absorption capacity and maintaining the defensive potential against the microbial and chemical challenges. It is suggested that the disruption of intestinal barrier integrity is generally accompanied by various GIT disorders including intestinal infections, necrotizing enterocolitis, and inflammatory bowel diseases. However, most of the studies support the fact that probiotics are very helpful in restoration of the gut barrier functions even after damage occurs, e.g., probiotic strain E. coli Nissle 1917 stimulates the function of junctional barrier after disruption induced by the enteropathogenic strain of E. coli in the T84 gut epithelial cell lines [35]. Probiotic microbes are ingested by microfold (M) cells to communicate with follicle-associated gut epithelial cells and dendritic cells that eventually initiate the responses mediated by $\mathrm{T}$ cells, $\mathrm{B}$ cells, and macrophages [36]. Another immunomodulatory mechanism regulated by probiotics is the activation of Toll-like receptors (TLRs). The other mechanisms include the restoration of mucosal surfaces and entrapment of pathogens by stimulating the mucus production. Further, specific probiotics can regulate both local and systemic immune responses [37]. The overall effects of probiotics on the intestinal functions are described in Figure 1.

\section{Desired Features for Probiotics}

The initial choice of probiotic strain for genetic modifications includes analysis of the following essential criteria: genotype and phenotype stability together with the stability of plasmids, protein or carbohydrate utilization patterns, bile or acid tolerance, growth as well as survival, antibiotic resistance patterns, intestinal epithelial adhesion characteristics, production of antimicrobials, ability to impede known pathogens, and immunogenicity [38]. Furthermore, a probiotic is additionally looked for the following features: gastric acid resistance, resistance to pancreatic enzymes (PE), colonization capacity, adherence to intestinal mucosal cells, and ability to produce substances against pathogens [39]. Probiotic encapsulation technology (PET) has emerged as an exciting field during the past decade that tends to stabilize the bacterial cells, therefore enhancing the viability during the production as well as storage. Further, this technology ensures the controlled and effective delivery of the bacterial strains to the gut with greater viability and protection in the acidic environment of the stomach. Hence, probiotics may exert better health benefits to the host in their viable state [40].

It is often recommended that the origin of the probiotics used for a human should be a human source, although some of the strains which are generally not confined to human sources can also be considered to be very effective, for example, Bifidobacterium animalis [41]. Probiotic attributes are not associated with the genre or species of the microbe, but with selected strains of a particular species [42].
Classification of the probiotic strains is essential to get the knowledge about the strain and its probiotic potential [43].

The survival of probiotic strains greatly depends on their location; therefore, probiotic strains need to colonize and proliferate at their specific sites. The adaptation of the probiotic strains to the host's intestine is the key step to handle the native microbiota and colonize a specific niche. Moreover, the probiotic strains should be acceptable by the immune system with no cellular or humoral immunity against that specific probiotic strain [44].

During the selection of probiotics, the key features and efficacy should be initially evaluated as well as a basic preliminary characterization in addition to the strain identity, taxonomy, and risk assessment using the standardized tests in both animal models and controlled studies within the target host. In vitro studies are commonly conducted although they are not predictive of in vivo functions. Moreover, the technological features should also be evaluated to know the capacity of strains regarding growth at a large scale and assimilation into the end product [45]. The selection criteria for probiotics are explained in Table 1.

\section{Recombinant Probiotics}

Although antibiotic treatment is the primary choice in the case of microbial attack, overuse, as well as misuse of antibiotics, results in the development of antimicrobial resistance [46]. Antibiotic treatment can remove useful microorganisms and results in the emergence of resistant microbial strains [47]. This has provoked the need to develop unique antimicrobial substances that sound riskless, environment-friendly, and active against antibiotic-resistant microbes [48]. In the previous decade, GM probiotics have been produced for the mucosal delivery of prophylactic and therapeutic including enzymes, DNA, cytokines, peptides, and allergens [49]. Oral engineered probiotics have many certain advantages such as stability, lower delivery cost, delivery of substances to mucosal surfaces, and increased shelf life.

\section{Applications of GM Probiotics}

6.1. GM Probiotics to Produce Antimicrobial Peptides. The emergence of antibiotic resistance among bacterial pathogens necessitates the use of alternative strategies for the management of infections. One of the potential alternative strategies to control multidrug-resistant pathogens includes the use of antimicrobial peptides (AMPs) which has been explored as an alternative method for effective control of multidrug-resistant (MDR) pathogens [50]. Few probiotics are known to produce various antimicrobial peptides; therefore, the probiotics can be used to produce and deliver these therapeutic antimicrobial peptides for the control of a specific pathogen within the GIT of the host [14]. So far, this technique is not much successful due to several limitations including the high cost of production, time-consuming, and the killing of the producing cells by their antimicrobial proteins. Moreover, the AMPs are degraded before reaching the target sites, i.e., intestine following the oral 


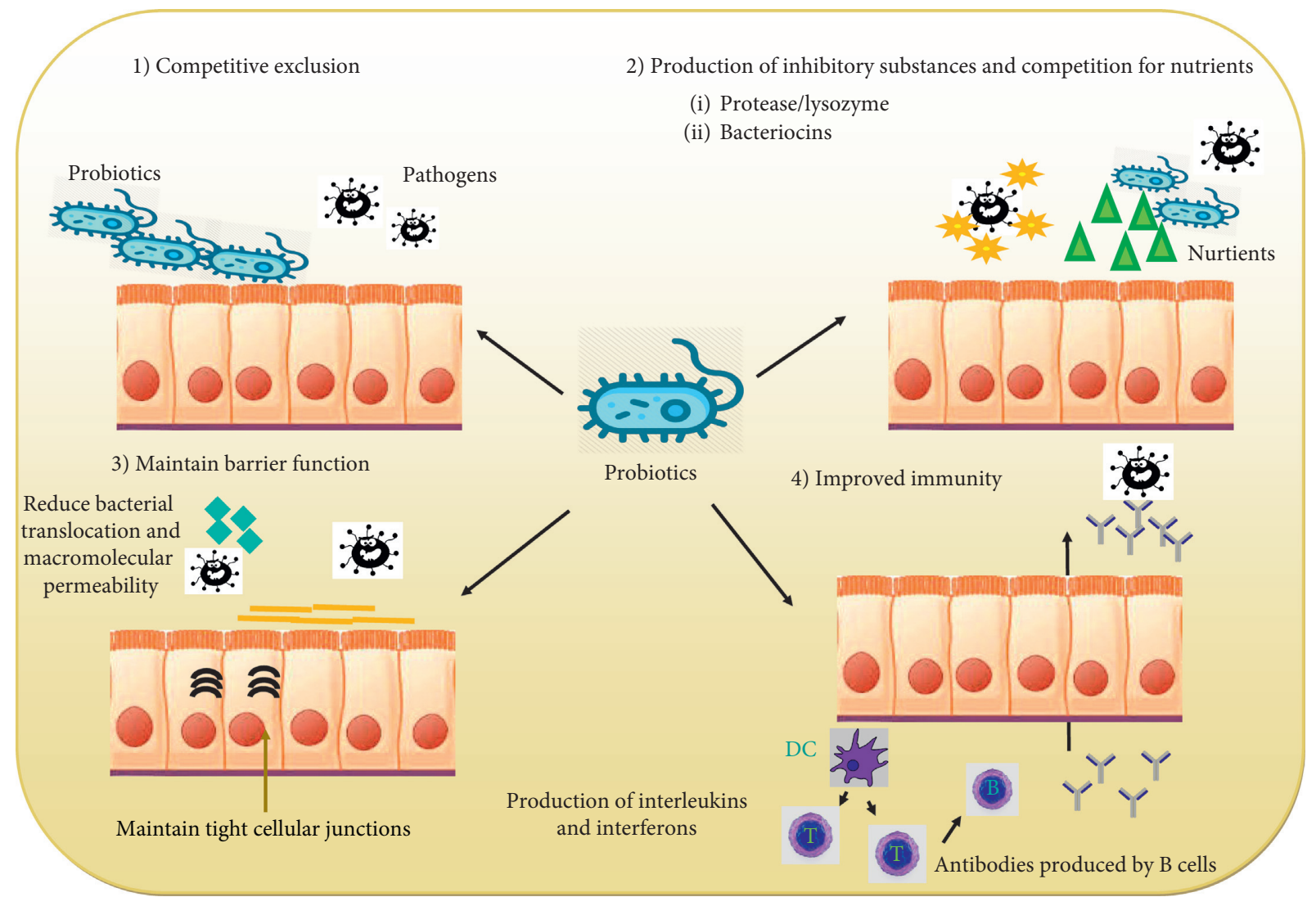

FIGURE 1: Role of probiotics in the amelioration of gut functions. (1) The probiotics could protect from pathogenic microbes in a diversified manner. (2) The probiotics can secrete the antimicrobial compounds and metabolites such as lactic acid, enzymes, and bacteriocins which can alter the $\mathrm{pH}$ that ultimately can inhibit the growth of pathogens. (3) The probiotics maintain the gut mucosal barriers, i.e., the chemical barriers such as antimicrobial peptides (AMPs), which can inhibit the invading microorganisms, and the physical barriers including cellular junction and the mucus layer that repel the invading microorganisms. (4) The alliance of microbiota with the immune system allows the maintenance of regulatory function involved in the conservation of tolerance to safer antigens and induction of protection to the pathogens.

administration. In the case of systemic administration, these AMPs are rapidly identified and eliminated by the immune system [51]. At present, different strategies are being used to use the probiotic strains to produce different AMPs resulting in a combination therapy so that the probiotic strains can offer the probiotic features accompanied by the production of the various AMPs [50].

A Lactococcus lactis strain IL1403 was engineered to express and secrete the AMPs with a considerable activity against the Gram-negative bacterial pathogens, i.e., Salmonella and E. coli strains. The AMPs such as alyteserin and A3APO were cloned into the L. lactis for the expression of these peptides. The resultant recombinant $L$. lactis strain was induced to secrete these peptides, and their effect on the viability and growth of Salmonella and E. coli was tested. Both pathogens were successfully inhibited, and the host strain, i.e., L. lactis, remained viable that showed that this recombinant system has the potential to be used as an alternative to the antibiotics to inhibit the Gram-negative bacterial pathogens $[25,52]$.

6.2. Stress Tolerance. The ability of the probiotic strain to tolerate more stress was one of the important factors to genetically modify the probiotics. Heat-shock proteins such as "GroES" and "GroEL" have been reported to play a key role in the persistence of bacterial species at all temperatures. The overexpression of these chaperones, i.e., GroES and GroEL in Lactobacillus paracasei NFBC338 was studied. The expression of such genes resulted in increased solvent resistance as well as improved thermotolerance in probiotic strains. Furthermore, the parent strain (nonadapted), stressadapted strain, and recombinant strain were compared for the survival following the exposure to thermal stress. The engineered probiotic strains survived about 54-fold compared to the nonadopted parent strain while 10-fold as compared with the stress-adapted strains [25].

Three major transport mechanisms or systems have been recognized in Listeria monocytogenes that are related to carnitine as well as betaine uptake. The first transport mechanism is named as Listerial betaine transport uptake system (BetL), in which genes that encode glycine betaine transporter are related to salt tolerance of Listerial species. The expression of the BetL system into Lactobacillus salivarius UCC118 probiotic strain was investigated by using another expression system, namely, a nisin-controlled system. It was noticed that probiotic resistance to many stresses is increased by using the BetL expression system [53]. These 
TABLe 1: Selection criteria of probiotics.

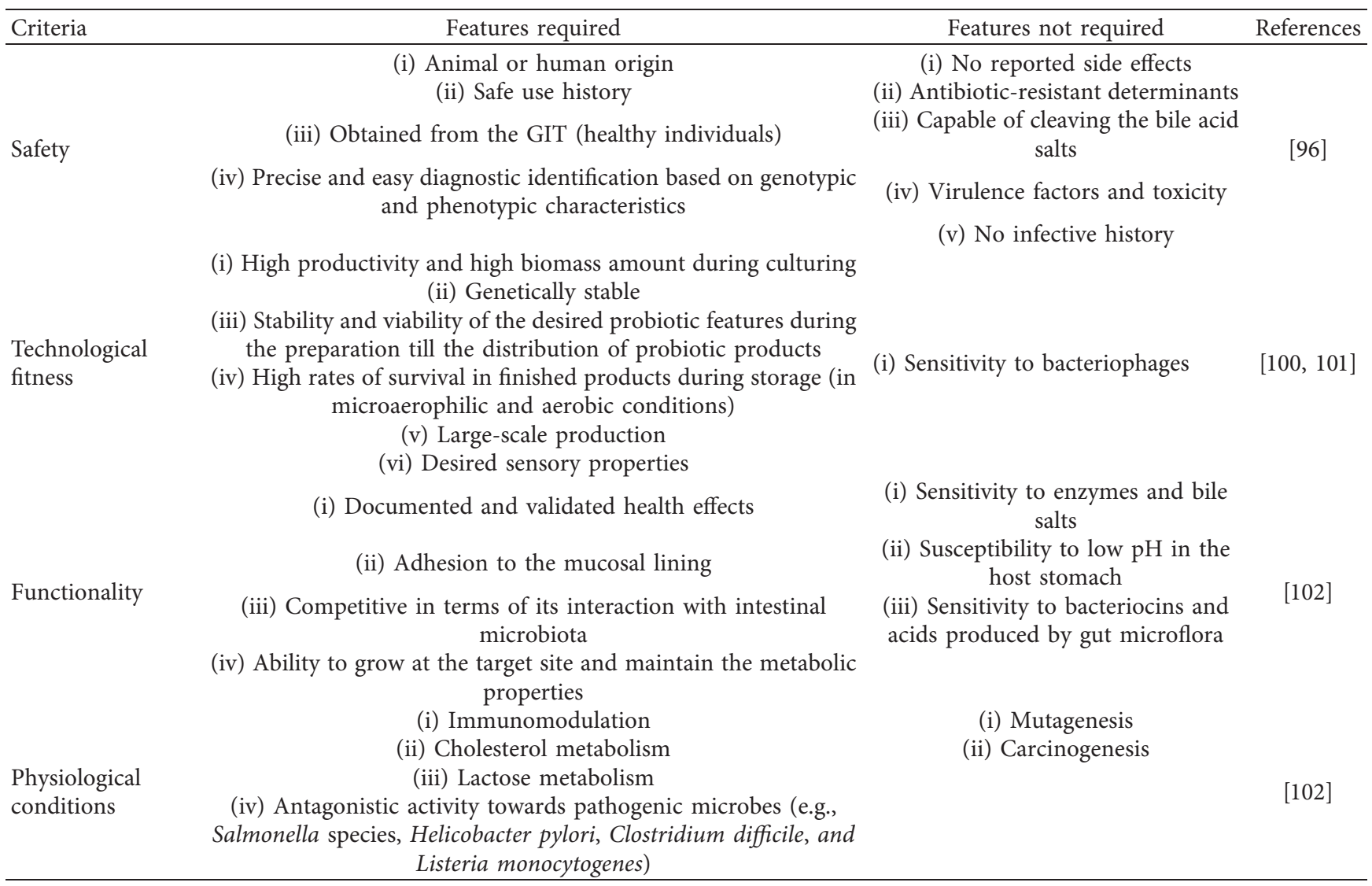

studies revealed promising approaches regarding the transfer of genes from pathogens to probiotics for the improvement of stress tolerance. Furthermore, scientific evaluation is quite essential to understand the benefits of these gene products through the study of risk-benefit analysis $[54,55]$.

6.3. Anticancer Therapeutics. The conventional anticancer treatments like chemotherapy and the increasing resistance to anticancer agents have stressed the need to switch on searching the alternative therapeutic approaches. In this situation, engineered probiotics can overcome these limitations by precisely targeting the tumor cells. Many of the bacterial species like clostridia, when injected either internally or externally to tumor cells, resulted in increased replication of bacteria inside tumor microenvironment and thus were found helpful to cope with cancers [56]. Salmonella typhimurium A1-R (GM tumor-targeting variant) can also be used as a replacement of previous strategies, namely, clostridial delivery systems for the treatment of malignant cancers [57]. The easier access to genetic manipulation strategies offers new opportunities to establish unique technologies both in cancer therapeutics and in cancer diagnosis [58, 59]. Nonpathogenic strains like E. coli Nissle 1917 can target and reproduce within the tumor cells as well as necrotic tissues [60]. The overall mechanisms of colonization and intratumoral relocation can be affected by the bacterial metabolome as well as by tumor microenvironment
[61]. This native tropism ability for certain cancers by microorganisms is considered as ideal for the safe delivery of new innovative therapeutic modalities which can save the patient from the potential side effects of drug-associated toxicity [62]. The approach could also help to treat primary as well as metastatic melanomas by using cancer colonizing facultative and obligate anaerobes such as Shigella, E. coli, and Clostridia strains, Bifidobacterium, Salmonella, and certain oncolytic viruses $[63,64]$. Since the mechanism of action of certain bacteria is not yet fully described, it could be quite easy to explore the mixture of microbes for treating various types of cancers [46].

6.4. Cognitive Health. A newly introduced probiotic group, i.e., psychobiotics that are able to produce neuroactive molecules such as serotonin and gamma-aminobutyric acid usually acts upon a gut-brain axis and is helpful for improving cognitive health [65]. Approximately 100 million nerve cells (from the gastrointestinal tract to the base of the brain) are connected through the vagus nerve; therefore, the signals released by intestinal microorganisms can influence several behavioral and physiological responses by these nerve cells. The in vivo and in vitro analysis proposed that intestinal microorganisms and metabolites may affect the development of the central nervous system (CNS) and regulate the stress responses and neural circuitry [66]. The murine model of infection has been studied for understanding the mechanism and immune-regulatory effects of 
Lactobacillus rhamnosus JB-1 bacterium [67]. The evidence strongly suggests that gut microbes can affect mental health and therefore endorses further testing to validate the relationship between intestinal microbes and human behavior as well as neurological disorders in near future $[68,69]$. Studies about intestinal microbiota-brain communications also suggest that the microbial-based therapeutic approaches may help in treating mood disorders [66]. For instance, the $\mathrm{LAB}$ can decrease the level of neurotoxic substances such as indoles, amines, and ammonia [70]. A randomized, placebocontrolled trial has shown that cognitive reactivity to sad mood was considerably reduced after using multispecies probiotics for a period of 4 weeks [71].

6.5. Feminine Health. Recurring UTI (urinary tract infections) among women has entailed the importance of preventive and management strategies of UTIs. Numerous strains of probiotics have great efficiency in the prevention of heterosexual transmission of viral infections [46, 72]. Clinical, experimental, and epidemiological studies have shown that normal vaginal microflora plays a protecting role or provides defense against the acquisition of microbial vaginosis and other sexually transmitted infections $[73,74]$. However, some studies disagree with the fact that probiotic use is helpful in preventing bacterial vaginosis, STDs (sexually transmitted diseases), and urinary tract infections $[11,72]$. The genitourinary and intestinal environment are usually best because they produce antagonists at mucosal lining but also colonize these areas and exert very potential homeostatic effects $[46,75]$. In this regard, the development of designer microbicides for preventing sexual transmission of HIV-1 could be the main target to control the AIDS epidemic worldwide [75]. It was suggested that the antiviral peptides produced by the probiotics can provide defense against HIV infection. HIV-gp41 hemolysin A peptide has been engineered by using E. coli strain, namely, Nissle 1917 $(\mathrm{EcN})$, which is helpful in inhibiting HIV infection. The secretion and growth of these antiviral peptides indicate that genetically modified probiotics can have anti-HIV properties [76].

6.6. Immunomodulation and Cytoprotection. The attenuated pathogens have been used as vaccines although the chance of reversion back to the virulent state exists particularly when injected into the immunocompromised subjects. This problem can be addressed using GM probiotics which can effectively carry the immunogenic substances to the mucosal cell surface [77]. Recombinant probiotic bacterial strains could act as an ideal vector as they are inherent to the host's mucosal surfaces, thus facilitating the contact between the immune system and antigen. Furthermore, intestinal colonization by probiotic bacteria continuously produces immunogenic molecules to activate humoral as well as cell-mediated immune responses [78]. Some probiotic bacteria have been manipulated as vaccine delivery agents against Streptococcus pneumonia, Salmonella Typhimurium, and Yersinia pseudotuberculosis infection $[79,80]$. The live attenuated vaccines for instance against rotavirus infection have been produced; however, they were found less effective due to a lack of strong mucosalassociated immune response. To stimulate a potent immune response, GM probiotic strain, i.e., L. paracasei ATCC 393 pLZ15 ${ }^{-}$, was developed which significantly decreases the viral load as well as reduces the severity of disease within the mouse model [81]. Moreover, it was observed that GM Lactococcus lactis NZ9000 expressing spike-protein VP8 from the rotavirus induces the formation of anti-VP8 antibodies with an increased mucosal IgA (systemic and intestinal levels) in a mouse model of infection [82]. Besides using the heterologous antigens, the use of cytokines can also help in immune stimulation, for example, the oral intake of IL-10 in the case of colitis reduces the inflammatory symptoms. Human interferon- $\beta$ (huIFN- $\beta$ ) is considered as immunomodulatory and thus causes the increased secretion of IL-10 expression; huIFN$\beta$ secreting $L$. plantarum NCIMB8826 also significantly reduces microbial colitis and inflammatory process $[83,84]$.

6.7. Regulation of Virulent Gene Expression. Pathogenic microbes regulate and control the expression of virulent genes through a specialized phenomenon called "quorum sensing." Disruption of this specialized sensing pathway can assist as a sustainable choice for disease prevention [85]. E. coli Nissle strain producing autoinducer molecule, i.e., cholera autoinducer 1 (CAI-1), which was previously shown to stop the production of virulence factors in the presence of other signaling molecules, i.e., autoinducer 2, decreased the expression of virulence genes as well as colonization of Vibrio cholerae in the intestine of an infant mouse model [86]. The in vivo studies using animal models for the study of genetically modified probiotic strains in various clinical conditions are summarized in Table 2.

\section{Safety Concerns regarding Genetically Modified Probiotics}

One of the major concerns of GM probiotic strain and their use in the clinical settings is their safety issues; therefore, it is necessary to screen the bacteria for its virulent traits as well as for their potential pathogenicity [87]. Probiotics are universally acknowledged due to their prohealth facets. Although many side effects like immune system hyperactivation, mutagenesis, and undesirable metabolic activities have also been reported, few studies also reported some intestinal side effects and increased the stimulation of the immune system in susceptible hosts [88]. Although the intrinsic antibiotic resistance is a desired attribute in probiotic bacteria for maintaining the microbial balance within the intestines, however, the transmission of these resistant genes may also cause serious threats in terms of the development of multidrug-resistant pathogenic strains $[89,90]$.

The exclusion of antibiotic-resistant genes is very essential to improve the safety of the probiotic. The approach was applied for a GM probiotic strain specifically 
TABLE 2: Example of genetically modified probiotics microorganisms for use as designer probiotics in animal models.

\begin{tabular}{|c|c|c|c|c|}
\hline Disease target & Microbial strain & Model & Outcome & Reference \\
\hline Cancer & Bacteroides ovatus D-6 & Mice & Increased levels of TNF- $\alpha$-specific IgG and IgM & [103] \\
\hline Intestinal inflammation & Bacteroides ovatus V975 & Mice & $\begin{array}{l}\text { Decreased the symptoms of DSS-induced colitis in a mice } \\
\text { colitis model }\end{array}$ & {$[103]$} \\
\hline $\begin{array}{l}\text { Clearance of infectious } \\
\text { agents }\end{array}$ & $\begin{array}{l}\text { Bacteroides acidifaciens } \\
\text { JCM } 10556(T)\end{array}$ & Mice & Increased gut IgA levels in gnotobiotic mice & {$[104]$} \\
\hline $\begin{array}{l}\text { IBD mainly (also eczema, } \\
\text { asthma, and type II diabetes) }\end{array}$ & $\begin{array}{l}\text { Faecalibacterium } \\
\text { prausnitzii }\end{array}$ & Mice & Colitis and other diseases were focused in mice model & {$[105-107]$} \\
\hline Inflammatory bowel disease & $\begin{array}{l}\text { Lactococcus lactis (food } \\
\text { grade strain) }\end{array}$ & Mice & $\begin{array}{c}\text { Protection of mice from T-cell transfer-induced } \\
\text { enterocolitis mediated by the LL-IL-27 mediated through } \\
\text { mucosal delivery }\end{array}$ & {$[108]$} \\
\hline Oral mucositis & L. lactis sAGX0085 & Hamster & $\begin{array}{l}\text { Improved repair of intestinal epithelial damage possibly to } \\
\text { occur during radiotherapy or chemotherapy-induced } \\
\text { mucositis in cancer patients }\end{array}$ & {$[93]$} \\
\hline Inflammatory diseases & Streptococcus gordonii & Mice & $\begin{array}{l}\text { Demonstration of full biological activity through RFVP/ } \\
\text { IL-RA in vitro; the recombinant strain was suggested to be } \\
\text { suitable for selective targeting of the mucosal surface as a } \\
\text { delivery system }\end{array}$ & [109] \\
\hline $\begin{array}{l}\text { Mainly inflammatory } \\
\text { diseases such as IBD }\end{array}$ & Lactococcus lactis & Mice & Medium: good evidence from IBD animal models & [110] \\
\hline
\end{tabular}

Lactobacillus which is of great importance and is widely used in industry to produce many useful metabolites [91]. The use of GM bacteria requires strict security and safety measures [90]. Since the GM probiotics have additional genes responsible for immunomodulation and antigenicity and also have the ability to affect the metabolic pathways, the safety testing should be precisely carried out [92, 93]. Other concerns about GM probiotics are related to the persistence and proliferation of these engineered probiotics in the external environment [94]. The engineered microbes have the potential to prevent and treat different human pathological situations; therefore, it is necessary to develop a stringent criterion for the evaluation of the safety of these strains both in vitro and in vivo. The biological containment system can be used for the prevention of distribution of genetic content to other microbes. Owing to the therapeutic safety of GM probiotics, it is indispensable to assess the risk, exposure determination, and safety issues in preventing the population from the unintended event of probiotic use [95-97].

\section{Regulatory Concerns for Probiotic Products}

It is quite important to have a special regulatory category for the probiotic products as the probiotics are delivered to the end-users through foods, infant formula, dietary or nutritional supplements, natural health products, and medical foods. It is imperative to know that the existing safety requirements for each product category vary with the geographical area. One of the most important facts is that the requirements for these nutritional or dietary supplements are different compared to conventional drugs. In case of drugs, the premarket approval for their safety is essential; however, for the nutritional or dietary supplements, it is still not required; for example, the dietary supplements in the United States even in the form of capsules or pills are not required to fulfill the same standards as implemented for the manufacturing and quality control testing of drugs [98]. It is recommended that the probiotic manufacturers are accountable for the assurance of the safety and suitability of probiotic products. The production of probiotic products in the United States is regulated by the Food and Drug Authority (FDA) to follow the good manufacturing practices for food and dietary supplements [99]. The premarket approval is not required for the dietary supplements in the United States; consequently, the end-users or the healthcare providers are not sure about the quality standards of the probiotic products and the safety of the product contents. However, the companies may opt for a third-party verification for the finished product $[98,99]$.

It is a need of the hour to establish the regulatory status of probiotics products on a global level to effectively address the probiotic issues including safety, efficacy, claims, and labels. The probiotic formulations shown to confer welldefined health benefits to the host must be allowed to describe clearly their specific health benefits with an effective surveillance system to monitor and evaluate the adverse events associated with these probiotic formulations and to monitor their long-term health benefits. Moreover, the prospects of GM probiotics targeting specific clinical conditions require a stringent safety policy to avert the spread of the strains in the environment and spreading of the genetic modifications.

\section{Conclusion and Future Prospects}

The public interest in probiotic bacteria is on the upswing, and researchers are bringing traditional therapy into translational approaches during the $21^{\text {st }}$ century. Intestinal microbes eventually affect all the major components of health management including drug metabolism and identification, toxicology, and establishment of innovative therapies. The combined benefits of GM probiotics, including their direct antagonistic response against pathogenic bacteria as well as the immunomodulatory potentials against 
cancers, pathogenic bacteria, and metabolic diseases, can be appraised. Even though the findings regarding the recombinant bacteria and their metabolites are inconsistent, the recombinant probiotics are foreseen as emerging therapeutics. Particularly, the combined therapeutics approach comprising of a repertoire of antimicrobial, immunomodulatory, and anti-inflammatory functions of recombinant probiotics could be helpful to cope with the infectious as well as metabolic disorders. Thus far, most studies have documented the benefits of engineered probiotics in animal models; however, the reports from some clinical trials in humans are quite encouraging. Nevertheless, the key challenges for these engineered probiotics remain the same, i.e., the selection of probiotic strains, optimum dose, and horizontal gene transfer from GM probiotics to other bacterial species. In brief, the GM probiotics are potent and innovative alternative therapeutics for the management of infections and metabolic diseases. The engineered probiotics may be helpful to restore the health with ease, efficiency, and site specificity and further research should explore human microbiota for the development of potential engineered strains as alternative therapeutics.

\section{Conflicts of Interest}

The authors declare that they have no conflicts of interest.

\section{References}

[1] M. Georgieva, L. Andonova, L. Peikova, and A. Zlatkov, "Probiotics-health benefits, classification, quality assurance and quality control-review," International Journal of Pharmaceutical Sciences, vol. 61, 2014.

[2] M. Khurshid, B. Aslam, M. A. Nisar et al., "Bacterial munch for infants: potential pediatric therapeutic interventions of probiotics," Future Microbiology, vol. 10, no. 11, pp. 1881-1895, 2015.

[3] A. A. Khan, M. Khurshid, S. Khan, and A. Alshamsan, "Gut microbiota and probiotics: current status and their role in cancer therapeutics," Drug Development Research, vol. 74, no. 6, pp. 365-375, 2013.

[4] C. Lacroix and S. Yildirim, "Fermentation technologies for the production of probiotics with high viability and functionality," Current Opinion in Biotechnology, vol. 18, no. 2, pp. 176-183, 2007.

[5] M. F. Iulietto, P. Sechi, E. Borgogni, and B. T. Cenci-Goga, "Meat spoilage: a critical review of a neglected alteration due to ropy slime producing bacteria," Italian Journal of Animal Science, vol. 14, no. 3, p. 4011, 2015.

[6] M. Sherid, S. Samo, S. Sulaiman, H. Husein, H. Sifuentes, and S. Sridhar, "Liver abscess and bacteremia caused by lactobacillus: role of probiotics? Case report and review of the literature," BMC Gastroenterology, vol. 16, no. 1, p. 138, 2016.

[7] K. Michalke, A. Schmidt, B. Huber et al., "Role of intestinal microbiota in transformation of bismuth and other metals and metalloids into volatile methyl and hydride derivatives in humans and mice," Applied and Environmental Microbiology, vol. 74, no. 10, pp. 3069-3075, 2008.

[8] J. Antikainen, V. Kuparinen, K. Lähteenmäki, and T. K. Korhonen, "Enolases from gram-positive bacterial pathogens and commensal lactobacilli share functional similarity in virulence-associated traits," FEMS Immunology \& Medical Microbiology, vol. 51, no. 3, pp. 526-534, 2007.

[9] C. L. Rohde, V. Bartolini, and N. Jones, "The use of probiotics in the prevention and treatment of antibiotic-associated diarrhea with special interest in Clostridium difficile-associated diarrhea," Nutrition in Clinical Practice, vol. 24, no. 1, pp. 33-40, 2009.

[10] B. Olle, "Medicines from microbiota," Nature Biotechnology, vol. 31, no. 4, pp. 309-315, 2013.

[11] A. Homayouni, P. Bastani, S. Ziyadi et al., "Effects of probiotics on the recurrence of bacterial vaginosis," Journal of Lower Genital Tract Disease, vol. 18, no. 1, pp. 79-86, 2014.

[12] M. S. R. Rajoka, H. M. Mehwish, H. Zhang et al., "Antibacterial and antioxidant activity of exopolysaccharide mediated silver nanoparticle synthesized by Lactobacillus brevis isolated from Chinese koumiss," Colloids and Surfaces B: Biointerfaces, 2019.

[13] M. S. R. Rajoka, H. M. Mehwish, H. Fang et al., "Characterization and anti-tumor activity of exopolysaccharide produced by Lactobacillus kefiri isolated from Chinese kefir grains," Journal of Functional Foods, vol. 63, Article ID 103588, 2019.

[14] M. A. R. Amalaradjou and A. K. Bhunia, "Bioengineered probiotics, a strategic approach to control enteric infections," Bioengineered, vol. 4, no. 6, pp. 379-387, 2013.

[15] L. Steidler, "Genetically engineered probiotics," Best Practice \& Research Clinical Gastroenterology, vol. 17, no. 5, pp. 861-876, 2003.

[16] K. J. Chua, W. C. Kwok, N. Aggarwal, T. Sun, and M. W. Chang, "Designer probiotics for the prevention and treatment of human diseases," Current Opinion in Chemical Biology, vol. 40, pp. 8-16, 2017.

[17] S. E. Evivie, G.-C. Huo, J. O. Igene, and X. Bian, "Some current applications, limitations and future perspectives of lactic acid bacteria as probiotics," Food \& Nutrition Research, vol. 61, no. 1, Article ID 1318034, 2017.

[18] A. A. Amara and A. Shibl, "Role of probiotics in health improvement, infection control and disease treatment and management," Saudi Pharmaceutical Journal, vol. 23, no. 2, pp. 107-114, 2015.

[19] M. J. Kullen and T. R. Klaenhammer, "Genetic modification of intestinal lactobacilli and bifidobacteria," Current Issues in Molecular Biology, vol. 2, no. 2, pp. 41-50, 2000.

[20] J. E. Boyington, B. Schoster, K. Remmes Martin, J. Shreffler, and L. F. Callahan, "Perceptions of individual and community environmental influences on fruit and vegetable intake, North Carolina, 2004," Preventing Chronic Disease, vol. 6, no. 1, p. A04, 2009.

[21] N. Tharmaraj and N. P. Shah, "Antimicrobial effects of probiotics against selected pathogenic and spoilage bacteria in cheese-based dips," International Food Research Journal, vol. 16, no. 1, pp. 261-276, 2009.

[22] S. Tejero-Sariñena, J. Barlow, A. Costabile, G. R. Gibson, and I. Rowland, "Antipathogenic activity of probiotics against salmonella typhimurium and Clostridium difficile in anaerobic batch culture systems: is it due to synergies in probiotic mixtures or the specificity of single strains?" Anaerobe, vol. 24, pp. 60-65, 2013.

[23] C. Aguilar, C. Vanegas, and B. Klotz, "Antagonistic effect of Lactobacillus strains against Escherichia coli and Listeria monocytogenes in milk," Journal of Dairy Research, vol. 78, no. 2, pp. 136-143, 2011.

[24] O. Karimi and A. S. Peña, "Indications and challenges of probiotics, prebiotics, and synbiotics in the management of 
arthralgias and spondyloarthropathies in inflammatory bowel disease," Journal of Clinical Gastroenterology, vol. 42, no. 3, pp. S136-S141, 2008.

[25] M. G. Mathipa and M. S. Thantsha, "Probiotic engineering: towards development of robust probiotic strains with enhanced functional properties and for targeted control of enteric pathogens," Gut Pathogens, vol. 9, no. 1, p. 28, 2017.

[26] N. P. Shah, "Functional cultures and health benefits," International Dairy Journal, vol. 17, no. 11, pp. 1262-1277, 2007.

[27] K. Kailasapathy and J. Chin, "Survival and therapeutic potential of probiotic organisms with reference to Lactobacillus acidophilus and Bifidobacterium spp," Immunology and Cell Biology, vol. 78, no. 1, pp. 80-88, 2000.

[28] E. P. Culligan, C. Hill, and R. D. Sleator, "Probiotics and gastrointestinal disease: successes, problems and future prospects," Gut Pathogens, vol. 1, no. 1, p. 19, 2009.

[29] M. P. Conte, S. Schippa, I. Zamboni et al., "Gut-associated bacterial microbiota in paediatric patients with inflammatory bowel disease," Gut, vol. 55, no. 12, pp. 1760-1767, 2006.

[30] S. J. Ott, M. Musfeldt, D. F. Wenderoth et al., "Reduction in diversity of the colonic mucosa associated bacterial microflora in patients with active inflammatory bowel disease," Gut, vol. 53, no. 5, pp. 685-693, 2004.

[31] S. C. J. De Keersmaecker, T. L. A. Verhoeven, J. Desair, K. Marchal, J. Vanderleyden, and I. N. Nagy, "Strong antimicrobial activity of Lactobacillus rhamnosus GG against Salmonella typhimuriumis due to accumulation of lactic acid," FEMS Microbiology Letters, vol. 259, no. 1, pp. 89-96, 2006.

[32] M. Shahid, B. Hussain, D. Riaz, M. Khurshid, M. Ismail, and M. Tariq, "Identification and partial characterization of potential probiotic lactic acid bacteria in freshwater Labeo rohita and Cirrhinus mrigala," Aquaculture Research, vol. 48, no. 4, pp. 1688-1698, 2017.

[33] M. Roselli, A. Finamore, M. S. Britti, and E. Mengheri, "Probiotic bacteria Bifidobacterium animalis MB5 and Lactobacillus rhamnosus GG protect intestinal Caco-2 cells from the inflammation-associated response induced by enterotoxigenic Escherichia coli K88," British Journal of Nutrition, vol. 95, no. 6, pp. 1177-1184, 2006.

[34] P. M. Sherman, K. C. Johnson-Henry, H. P. Yeung, P. S. C. Ngo, J. Goulet, and T. A. Tompkins, "Probiotics reduce enterohemorrhagic Escherichia coli O157:H7- and enteropathogenic E. coli O127:H6-induced changes in polarized T84 epithelial cell monolayers by reducing bacterial adhesion and cytoskeletal rearrangements," Infection and Immunity, vol. 73, no. 8, pp. 5183-5188, 2005.

[35] A. A. Zyrek, C. Cichon, S. Helms, C. Enders, U. Sonnenborn, and M. A. Schmidt, "Molecular mechanisms underlying the probiotic effects of Escherichia coli Nissle 1917 involve ZO-2 and PKC? Redistribution resulting in tight junction and epithelial barrier repair," Cellular Microbiology, vol. 9, no. 3, pp. 804-816, 2007.

[36] P. Winkler, D. Ghadimi, J. Schrezenmeir, and J.-P. Kraehenbuhl, "Molecular and cellular basis of microflora-host interactions," The Journal of Nutrition, vol. 137, no. 3, pp. 756s-772s, 2007.

[37] A. J. Macpherson and T. Uhr, "Induction of protective IgA by intestinal dendritic cells carrying commensal bacteria," Science, vol. 303, no. 5664, pp. 1662-1665, 2004.

[38] E. B.-M. Daliri and B. H. Lee, "New perspectives on probiotics in health and disease," Food Science and Human Wellness, vol. 4, no. 2, pp. 56-65, 2015.
[39] T. Ayichew, A. Belete, T. Alebachew, H. Tsehaye, and H. Berhanu, "Bacterial probiotics their importances and limitations: a review," Journal of Nutrition and Health Sciences, vol. 4, no. 2, p. 202, 2017.

[40] G. K. Gbassi and T. Vandamme, "Probiotic encapsulation technology: from microencapsulation to release into the gut," Pharmaceutics, vol. 4, no. 1, pp. 149-163, 2012.

[41] M. E. Sanders, "Summary of probiotic activities of Bifidobacterium lactis HN019," Journal of Clinical Gastroenterology, vol. 40, no. 9, pp. 776-783, 2006.

[42] C. Hill, F. Guarner, G. Reid et al., "The International Scientific Association for Probiotics and Prebiotics consensus statement on the scope and appropriate use of the term probiotic," Nature Reviews Gastroenterology \& Hepatology, vol. 11, no. 8, pp. 506-514, 2014.

[43] M. Caselli, D. Vaira, F. Cassol et al., "Recombinant Probiotics and their potential in human health," International Journal of Probiotics \& Prebiotics, vol. 7, no. 2, 2012.

[44] S. Fijan, "Microorganisms with claimed probiotic properties: an overview of recent literature," International Journal of Environmental Research and Public Health, vol. 11, no. 5, pp. 4745-4767, 2014.

[45] M. E. Sanders, "Probiotics: definition, sources, selection, and uses," Clinical infectious Diseases, vol. 46, no. 2, pp. S58-S61, 2008.

[46] B. Singh, G. Mal, D. Bharti et al., "Probiotics in female reproductive health: paradigms, prospects and challenges," Current Women's Health Reviews, vol. 9, no. 4, pp. 235-244, 2013.

[47] I. Y. Hwang, E. Koh, A. Wong et al., "Engineered probiotic Escherichia coli can eliminate and prevent Pseudomonas aeruginosa gut infection in animal models," Nature Communications, vol. 8, p. 15028, 2017.

[48] L. Steidler, W. Hans, L. Schotte et al., "Treatment of murine colitis by Lactococcus lactis secreting interleukin-10," Science, vol. 289, no. 5483, pp. 1352-1355, 2000.

[49] J. Wells, "Mucosal vaccination and therapy with genetically modified lactic acid bacteria," Annual Review of Food Science and Technology, vol. 2, no. 1, pp. 423-445, 2011.

[50] S. M. Mandal, O. N. Silva, and O. L. Franco, "Recombinant probiotics with antimicrobial peptides: a dual strategy to improve immune response in immunocompromised patients," Drug Discovery Today, vol. 19, no. 8, pp. 1045-1050, 2014.

[51] P. Kumar, J. N. Kizhakkedathu, and S. K. Straus, "Antimicrobial peptides: diversity, mechanism of action and strategies to improve the activity and biocompatibility in vivo," Biomolecules, vol. 8, no. 1, p. 4, 2018.

[52] K. Volzing, J. Borrero, M. J. Sadowsky, and Y. N. Kaznessis, "Antimicrobial peptides targeting gram-negative pathogens, produced and delivered by lactic acid bacteria," ACS Synthetic Biology, vol. 2, no. 11, pp. 643-650, 2013.

[53] V. M. Sheehan, R. D. Sleator, G. F. Fitzgerald, and C. Hill, "Heterologous expression of BetL, a betaine uptake system, enhances the stress tolerance of Lactobacillus salivarius UCC118," Applied and Environmental Microbiology, vol. 72, no. 3, pp. 2170-2177, 2006.

[54] R. D. Sleator and C. Hill, "Bioengineered bugs'-a pathobiotechnology approach to probiotic research and applications," Medical Hypotheses, vol. 70, no. 1, pp. 167-169, 2008.

[55] Z. Li, X. Pei, S. Yin, X. Lang, X. Zhao, and G.-Z. Qu, "Plant hormone treatments to alleviate the effects of salt stress on germination of Betula platyphylla seeds," Journal of Forestry Research, vol. 30, no. 3, pp. 779-787, 2019. 
[56] J. T. Heap, J. Theys, M. Ehsaan et al., "Spores of Clostridium engineered for clinical efficacy and safety cause regression and cure of tumors in vivo," Oncotarget, vol. 5, no. 7, pp. 1761-1769, 2014.

[57] R. M. Hoffman, "Back to the future: are tumor-targeting bacteria the next-generation cancer therapy?" Methods in Molecular Biology, vol. 1317, pp. 239-260, 2015.

[58] J. T. Panteli, B. A. Forkus, N. Van Dessel, and N. S. Forbes, "Genetically modified bacteria as a tool to detect microscopic solid tumor masses with triggered release of a recombinant biomarker," Integrative Biology, vol. 7, no. 4, pp. 423-434, 2015.

[59] N. Van Dessel, C. A. Swofford, and N. S. Forbes, "Potent and tumor specific: arming bacteria with therapeutic proteins," Therapeutic Delivery, vol. 6, no. 3, pp. 385-399, 2015.

[60] B. Brichacek, L. A. Lagenaur, P. P. Lee, D. Venzon, and D. H. Hamer, "In vivo evaluation of safety and toxicity of a Lactobacillus jensenii producing modified cyanovirin- $\mathrm{N}$ in a rhesus macaque vaginal challenge model," PloS One, vol. 8, no. 11, Article ID e78817, 2013.

[61] J. Stritzker, S. Weibel, C. Seubert et al., "Enterobacterial tumor colonization in mice depends on bacterial metabolism and macrophages but is independent of chemotaxis and motility," International Journal of Medical Microbiology, vol. 300, no. 7, pp. 449-456, 2010.

[62] D. Andreu and M. Torrent, "Prediction of bioactive peptides using artificial neural networks," Methods in Molecular Biology, vol. 1260, pp. 101-118, 2015.

[63] J. Stritzker and A. A. Szalay, "Single-agent combinatorial cancer therapy," Proceedings of the National Academy of Sciences, vol. 110, no. 21, pp. 8325-8326, 2013.

[64] W.-W. Chang and C.-H. Lee, "Salmonella as an innovative therapeutic antitumor agent," International Journal of Molecular Sciences, vol. 15, no. 8, pp. 14546-14554, 2014.

[65] T. G. Dinan, C. Stanton, and J. F. Cryan, "Psychobiotics: a novel class of psychotropic," Biological Psychiatry, vol. 74, no. 10, pp. 720-726, 2013.

[66] P. Forsythe, W. A. Kunze, and J. Bienenstock, "On communication between gut microbes and the brain," Current Opinion in Gastroenterology, vol. 28, no. 6, pp. 557-562, 2012.

[67] K. Al-Nedawi, M. F. Mian, N. Hossain et al., "Gut commensal microvesicles reproduce parent bacterial signals to host immune and enteric nervous systems," FASEB Journal, vol. 29, no. 2, pp. 684-695, 2015.

[68] S. Reardon, "Microbiome therapy gains market traction," Nature, vol. 509, no. 7500, pp. 269-270, 2014.

[69] S. Dash, G. Clarke, M. Berk, and F. N. Jacka, "The gut microbiome and diet in psychiatry," Current Opinion in Psychiatry, vol. 28, no. 1, pp. 1-6, 2015.

[70] A. Ramezani, Z. A. Massy, B. Meijers, P. Evenepoel, R. Vanholder, and D. S. Raj, "Role of the gut microbiome in uremia: a potential therapeutic target," American Journal of Kidney Diseases, vol. 67, no. 3, pp. 483-498, 2016.

[71] L. Steenbergen, R. Sellaro, S. van Hemert, J. A. Bosch, and L. S. Colzato, "A randomized controlled trial to test the effect of multispecies probiotics on cognitive reactivity to sad mood," Brain, Behavior, and Immunity, vol. 48, pp. 258-264, 2015.

[72] G. Reid, P. Brigidi, J. P. Burton et al., "Microbes central to human reproduction," American Journal of Reproductive Immunology, vol. 73, no. 1, pp. 1-11, 2015.

[73] R. M. Brotman, M. D. Shardell, P. Gajer et al., "Interplay between the temporal dynamics of the vaginal microbiota and human papillomavirus detection," Journal of Infectious Diseases, vol. 210, no. 11, pp. 1723-1733, 2014.

[74] A. Buvé, V. Jespers, T. Crucitti, and R. N. Fichorova, "The vaginal microbiota and susceptibility to HIV," Aids, vol. 28, no. 16, pp. 2333-2344, 2014.

[75] L. Vangelista, M. Secchi, X. Liu et al., "Engineering of Lactobacillus jensenii to secrete RANTES and a CCR5 antagonist analogue as live HIV-1 blockers," Antimicrobial Agents and Chemotherapy, vol. 54, no. 7, pp. 2994-3001, 2010.

[76] S. Rao, S. Hu, L. McHugh et al., "Toward a live microbial microbicide for HIV: commensal bacteria secreting an HIV fusion inhibitor peptide," Proceedings of the National Academy of Sciences, vol. 102, no. 34, pp. 11993-11998, 2005.

[77] S. Tarahomjoo, "Development of vaccine delivery vehicles based on lactic acid bacteria," Molecular Biotechnology, vol. 51, no. 2, pp. 183-199, 2012.

[78] J. M. Wells and A. Mercenier, "Mucosal delivery of therapeutic and prophylactic molecules using lactic acid bacteria," Nature Reviews Microbiology, vol. 6, no. 5, pp. 349-362, 2008.

[79] L. Hernani Mde, P. C. Ferreira, D. M. Ferreira, E. N. Miyaji, P. L. Ho, and M. L. Oliveira, "Nasal immunization of mice with Lactobacillus casei expressing the pneumococcal surface protein $\mathrm{C}$ primes the immune system and decreases pneumococcal nasopharyngeal colonization in mice," FEMS Immunology and Medical Microbiology, vol. 62, no. 3, pp. 263-272, 2011.

[80] A. Kajikawa, L. Zhang, J. Long et al., "Construction and immunological evaluation of dual cell surface display of HIV-1 gag and Salmonella enterica serovar typhimurium FliC in Lactobacillus acidophilus for vaccine delivery," Clinical and Vaccine Immunology, vol. 19, no. 9, pp. 13741381, 2012.

[81] N. Pant, A. Hultberg, Y. Zhao et al., "Lactobacilli expressing variable domain of llama heavy-chain antibody fragments (lactobodies) confer protection against rotavirus-induced diarrhea," The Journal of Infectious Diseases, vol. 194, no. 11, pp. 1580-1588, 2006.

[82] B. Marelli, A. R. Perez, C. Banchio, D. de Mendoza, and C. Magni, "Oral immunization with live Lactococcus lactis expressing rotavirus VP8* subunit induces specific immune response in mice," Journal of Virological Methods, vol. 175, no. 1, pp. 28-37, 2011.

[83] L. Fredriksen, C. R. Kleiveland, L. T. Olsen Hult et al., "Surface display of N-terminally anchored invasin by lactobacillus plantarum activates NF- $\mathrm{BB}$ in monocytes," Applied and Environmental Microbiology, vol. 78, no. 16, pp. 58645871, 2012.

[84] Z. Wang, Q. Yu, J. Gao, and Q. Yang, "Mucosal and systemic immune responses induced by recombinant Lactobacillus spp. expressing the hemagglutinin of the avian influenza virus H5N1," Clinical and Vaccine Immunology, vol. 19, no. 2, pp. 174-179, 2012.

[85] C. M. Waters and B. L. Bassler, "Quorum sensing: cell-to-cell communication in bacteria," Annual Review of Cell and Developmental Biology, vol. 21, pp. 319-346, 2005.

[86] F. Duan and J. C. March, "Engineered bacterial communication prevents Vibrio cholerae virulence in an infant mouse model," Proceedings of the National Academy of Sciences, vol. 107, no. 25, pp. 11260-11264, 2010.

[87] L. Dicks and M. Botes, "Probiotic lactic acid bacteria in the gastro-intestinal tract: health benefits, safety and mode of action," Beneficial Microbes, vol. 1, no. 1, pp. 11-29, 2010. 
[88] A. Rosander, E. Connolly, and S. Roos, "Removal of antibiotic resistance gene-carrying plasmids from Lactobacillus reuteri ATCC 55730 and characterization of the resulting daughter strain, L. Reuteri DSM 17938," Applied and Environmental Microbiology, vol. 74, no. 19, pp. 6032-6040, 2008.

[89] E. Broaders, C. G. M. Gahan, and J. R. Marchesi, "Mobile genetic elements of the human gastrointestinal tract," Gut Microbes, vol. 4, no. 4, pp. 271-280, 2013.

[90] A. Wong, D. Y. S. Ngu, L. A. Dan, A. Ooi, and R. L. H. Lim, "Detection of antibiotic resistance in probiotics of dietary supplements," Nutrition Journal, vol. 14, no. 1, p. 95, 2015.

[91] A. J. Rovner, A. D. Haimovich, S. R. Katz et al., "Recoded organisms engineered to depend on synthetic amino acids," Nature, vol. 518, no. 7537, pp. 89-93, 2015.

[92] E. A. Eloe-Fadrosh and D. A. Rasko, "The human microbiome: from symbiosis to pathogenesis," Annual Review of Medicine, vol. 64, no. 1, pp. 145-163, 2013.

[93] S. Caluwaerts, K. Vandenbroucke, L. Steidler et al., "AG013, a mouth rinse formulation of Lactococcus lactis secreting human Trefoil Factor 1, provides a safe and efficacious therapeutic tool for treating oral mucositis," Oral Oncology, vol. 46, no. 7, pp. 564-570, 2010.

[94] L. Steidler, S. Neirynck, N. Huyghebaert et al., "Biological containment of genetically modified Lactococcus lactis for intestinal delivery of human interleukin 10," Nature Biotechnology, vol. 21, no. 7, pp. 785-789, 2003.

[95] L. Torres, A. Krüger, E. Csibra, E. Gianni, and V. B. Pinheiro, "Synthetic biology approaches to biological containment: pre-emptively tackling potential risks," Essays in Biochemistry, vol. 60, no. 4, pp. 393-410, 2016.

[96] M. E. Sanders, L. M. A. Akkermans, D. Haller et al., "Safety assessment of probiotics for human use," Gut Microbes, vol. 1, no. 3, pp. 164-185, 2010.

[97] F. E. Ahmed, "Genetically modified probiotics in foods," Trends in Biotechnology, vol. 21, no. 11, pp. 491-497, 2003.

[98] M. E. Sanders, D. J. Merenstein, A. C. Ouwehand et al., "Probiotic use in at-risk populations," Journal of the American Pharmacists Association, vol. 56, no. 6, pp. 680686, 2016.

[99] S. A. Jackson, J. L. Schoeni, C. Vegge et al., "Improving enduser trust in the quality of commercial probiotic products," Frontiers in Microbiology, vol. 10, p. 739, 2019.

[100] R. Nagpal, A. Kumar, M. Kumar, P. V. Behare, S. Jain, and H. Yadav, "Probiotics, their health benefits and applications for developing healthier foods: a review," FEMS Microbiology Letters, vol. 334, no. 1, pp. 1-15, 2012.

[101] V. Nagarajan, M. Peng, Z. Tabashsum, S. Salaheen, J. Padilla, and D. Biswas, "Antimicrobial effect and probiotic potential of phage resistant lactobacillus plantarum and its interactions with zoonotic bacterial pathogens," Foods, vol. 8, no. 6, p. 194, 2019.

[102] P. Markowiak and K. Śliżewska, "Effects of probiotics, prebiotics, and synbiotics on human health," Nutrients, vol. 9, no. 9, 2017.

[103] P. Ulsemer, G. Henderson, K. Toutounian et al., "Specific humoral immune response to the Thomsen-Friedenreich tumor antigen (CD176) in mice after vaccination with the commensal bacterium bacteroides ovatus D-6," Cancer Immunology, Immunotherapy, vol. 62, no. 5, pp. 875-887, 2013.

[104] T. Yanagibashi, A. Hosono, A. Oyama et al., "IgA production in the large intestine is modulated by a different mechanism than in the small intestine: Bacteroides acidifaciens promotes
IgA production in the large intestine by inducing germinal center formation and increasing the number of IgA+ B cells," Immunobiology, vol. 218, no. 4, pp. 645-651, 2013.

[105] O. Rossi, L. A. van Berkel, F. Chain et al., "Faecalibacterium prausnitzii A2-165 has a high capacity to induce IL-10 in human and murine dendritic cells and modulates $\mathrm{T}$ cell responses," Scientific Reports, vol. 6, Article ID 18507, 2016.

[106] K. Simonyte Sjödin, L. Vidman, P. Rydén, and C. E. West, "Emerging evidence of the role of gut microbiota in the development of allergic diseases," Current Opinion in Allergy and Clinical Immunology, vol. 16, no. 4, pp. 390-395, 2016.

[107] H. Song, Y. Yoo, J. Hwang, Y.-C. Na, and H. S. Kim, "Faecalibacterium prausnitzii subspecies-level dysbiosis in the human gut microbiome underlying atopic dermatitis," Journal of Allergy and Clinical Immunology, vol. 137, no. 3, pp. 852-860, 2016.

[108] M. L. Hanson, J. A. Hixon, W. Li et al., "Oral delivery of IL-27 recombinant bacteria attenuates immune colitis in mice," Gastroenterology, vol. 146, no. 1, pp. 210.e213-221.e213, 2014.

[109] S. Ricci, G. Macchia, P. Ruggiero et al., "In vivo mucosal delivery of bioactive human interleukin 1 receptor antagonist produced by Streptococcus gordonii," BMC Biotechnology, vol. 3, no. 1, p. 15, 2003.

[110] J. P. Motta, L. G. Bermudez-Humaran, C. Deraison et al., "Food-grade bacteria expressing elafin protect against inflammation and restore colon homeostasis," Science Translational Medicine, vol. 4, no. 158, Article ID 158ra144, 2012. 